\title{
REGENERAÇÃO NATURAL EM UM REMANESCENTE DE CAATINGA SOB DIFE- RENTES NÍVEIS DE PERTURBAÇÃO, NO AGRESTE PARAIBANO
}

\author{
Israel Marinho Pereira ${ }^{1}$ \\ Leonaldo Alves de Andrade ${ }^{2}$ \\ José Ronaldo M. Costa ${ }^{1}$ \\ José Marcelo Dias ${ }^{1}$
}

Recebido em 17/12/99. Aceito em 15/09/01.

\begin{abstract}
RESUMO - (Regeneração natural em um remanescente de Caatinga sob diferentes níveis de perturbação, no agreste paraibano) $\mathrm{O}$ presente trabalho foi desenvolvido em um remanescente de caatinga hipoxerófila localizado na fronteira dos Municípios de Areia e Remígio-PB, com o objetivo de estudar o comportamento da regeneração natural em ambientes com diferentes níveis de perturbação antrópica. Foram selecionados três ambientes (I, II e III), ordenados em níveis crescentes de perturbação, tendo sido plotadas 5 unidades amostrais em cada um deles. Foram estabelecidas quatro classes de tamanho para estratificação dos indivíduos a serem estudados, conforme descritas a seguir: classe 1 - indivíduos com altura de 0,10 a $0,29 \mathrm{~m}$; classe 2 - indivíduos com altura de 0,30 a $1,49 \mathrm{~m}$; classe 3 - indivíduos com altura de 1,5 a $3 \mathrm{~m}$ e classe 4 - indivíduos com altura superior a $3 \mathrm{~m}$, mas que apresentavam o diâmetro na base inferior a três $\mathrm{cm}$. A densidade total foi da ordem de 5000 indivíduos ha ${ }^{-1}$, tendo sido identificadas 26 espécies arbóreas, pertencentes a 17 famílias. As famílias Euphorbiaceae e Mimosaceae detiveram aproximadamente $60 \%$ dos indivíduos amostrados. Nos ambientes I e II a espécie que apresentou maior regeneração natural foi Croton sonderianus Muell Arg., e no ambiente III, Thiloa glaucocarpa (Mart.) Eichl. A maioria dos indivíduos encontrava-se nas classes de menor tamanho, sendo que apenas o ambiente III, o mais conservado, apresentou indivíduos nas quatro classes de tamanho. Os maiores impactos da intervenção antrópica foram detectados no ambiente I, e os menores no ambiente III, refletidos a partir da riqueza florística, da dominância e da distribuição da plantas nas classes de tamanho previamente definidas.
\end{abstract}

Palavras-chaves - Regeneração Natural, Ecossistema de Caatinga, Perturbação Antrópica.

ABSTRACT - (Natural regeneration in a Caatinga fragment under different disturbance levels) This work was carried out on the border between the municipalities of Areia and Remígio, in the state of Paraíba, where some remnants of hypoxerophytic caatinga were encountered. The objective was to observe modes of natural regeneration in areas with different levels of anthropogenic disturbance. Three areas (I, II, III) with decreasing levels of disturbance were chosen. Five sample units from each area were chosen for plotting. Four size classes were established in order to stratify the specimens under study: class 1 - specimens ranging from 0.10 to $0.29 \mathrm{~m}$ in height; class 2 - specimens ranging from 0.30 to $1.49 \mathrm{~m}$ in height; class 3 - specimens ranging from 1.5 to $3 \mathrm{~m}$

\footnotetext{
${ }^{1}$ Mestres em Produção Vegetal - UFPB/CCA - Areia - PB

${ }^{2}$ Professor Adjunto UFPB/CCA/DF - Areia. CEP: 58.397-000. E-mail: landrade@cca.ufpb.br
} 
in height, and class 4 - specimens more than $3 \mathrm{~m}$ tall, but having a diameter of less than $3 \mathrm{~cm}$ at the base. The total density was in the range of 5000 specimens per ha ${ }^{-1}$. Twenty-six species of trees belonging to seventeen families were identified. $60 \%$ of the samples belonged to the Euphorbiaceae and Mimosaceae families. Croton sonderianus Muell. Arg. demonstrated the highest rate of natural regeneration in regions I and II, while in region III, the species with the highest rate of natural regeneration was Thiloa glaucocarpa (Mart.) Eichl. Most of the trees belonged to the smaller size categories. Only area III, the best preserved of all, contained specimens belonging to all four size categories. The greatest impact due to anthropogenic disturbances was detected in area I. The least impact was observed in area III, as proved by the richness of the forest and the predominance and distribution of plants in the previously defined size categories.

Key words - Natural regeneration, caatinga, anthropogenic disturbance, dry forest.

\section{Introdução}

$\mathrm{Na}$ tentativa de classificar e melhor entender os trópicos semi-áridos, inúmeros trabalhos têm sido desenvolvidos abordando as características físicas dessas regiões. Neste particular, o semi-árido do Nordeste brasileiro é relativamente bem conhecido, principalmente no que se refere aos seus limites territoriais e suas particularidades climáticas (Andrade et al., 1999; Reddy, 1983; Mcboyle, 1973; Nimer, 1972).

Constata-se, porém, uma grande escassez de informações no que se refere à dinâmica biológica dos ecossistemas aí inseridos. A vegetação predominante no semi-árido nordestino é um complexo genericamente denominado de caatinga, cuja classificação nem sempre é fácil.

As caatingas, em geral, são caracterizadas como formações arbóreo-arbustivas, hierarquizadas em diversas tipologias, muitas das quais ainda são praticamente desconhecidas do ponto de vista ecológico. Numa tentativa de adaptar a classificação da vegetação brasileira a um sistema universal, a caatinga nordestina foi recentemente classificada como 'savana estépica' (BRASIL, 1992).

Constata-se, porém, que, devido à grande extensão territorial que ocupa e os diferentes ambientes em que pode ser encontrada, a caatinga encerra uma enorme variabilidade de faciações fitogeográficas evidenciadas, principalmente, pelas diferenças fisionômicas, densidades, composição de espécies e aspectos fenológicos (Sampaio, 1995; Sampaio et al., 1993; Rodal et al., 1992; Andrade-Lima, 1981; Sou- sa, 1979). A carência de informações sobre a vegetação de caatinga se torna muito evidente quando se procura dados relativos, por exemplo, à estrutura fitossociológica, à dinâmica de populações, aos processos de sucessão ecológica e de regeneração natural dos ecossistemas aí encontrados (Araújo Filho, 1996; IBAMA, 1992).

Não obstante o desconhecimento do bioma, a caatinga vem sendo sistematicamente devastada, sem que se tenha conhecimentos profundos sobre aspectos relevantes da mesma. A eliminação sistemática da cobertura vegetal e o uso indevido das terras têm acarretado graves problemas ambientais no semi-árido nordestino, entre os quais se destacam a redução da biodiversidade, a degradação dos solos, o comprometimento dos sistemas produtivos e a desertificação de extensas áreas na maioria dos Estados que compõem a região (BRASIL, 1995; BRASIL, 1991; Japan, 1990; Dregne, 1986; Ab' Saber, 1977).

A exploração racional de qualquer ecossistema só pode ser planejada a partir do conhecimento de suas dinâmicas biológicas. No que se refere ao componente vegetação, torna-se imperativo conhecer, por exemplo, como se dão os processos de regeneração natural diante das perturbações antrópicas. Embora os estudos sobre regeneração natural de vegetação nativa não sejam recentes (Daubenmire, 1968), não existe consenso sobre o que melhor define essa expressão, nem, tão pouco, um conceito que contemple todas as situações encontradas nos diversos ecossistemas tropicais. A compreensão das di- 
nâmicas de regeneração natural em ecossistemas florestais possibilita que sejam feitas estimativas de parâmetros populacionais, imprescindíveis para a consecução do manejo florestal sustentado (Albuquerque, 1999; Drumond et al., 1996; Calegario et al., 1993).

Neste trabalho, teve-se como objetivo estudar a regeneração natural em ambientes de caatinga, sob diferentes níveis de perturbação antrópica, com vistas a oferecer subsídios técnicos às ações voltadas para a conservação e a recuperação do referido ecossistema.

\section{Material e Métodos}

O presente trabalho foi realizado, no período de setembro de 1998 a fevereiro de 1999 , em um remanescente de caatinga de aproximadamente 60 ha, localizado na Fazenda São Bento (latitude 6 $6^{\circ} 52$ ' $52^{\prime \prime} \mathrm{S}$; longitude $35^{\circ} 47^{\prime} 41^{\prime \prime} \mathrm{W}$; altitude $590 \mathrm{~m}$ ), na fronteira dos Municípios de Areia, Arara e Remígio no Agreste do Estado da Paraíba, Nordeste do Brasil. O agreste é uma microrregião que compreende a zona de transição, situada entre o litoral úmido e o sertão semiárido. Abrange áreas planas e superfícies elevadas da porção oriental do planalto da Borborema, onde se vê a transição entre os brejos úmidos e o sertão das caatingas (PNUD/FAO/IBAMA, 1994). O solo foi classificado como Regossolo distrófico, arenoso e mediamente raso, descrito como interpartes da zona da Borborema Ocidental, fase caatinga hipoxerófila, relevo plano e suave ondulado (BRASIL, 1971). Segundo o mesmo autor, o clima da área estudada é do tipo As' (quente e úmido) de Köppen, com pluviosidade média anual de $700 \mathrm{~mm}$, e déficit hídrico acentuado durante quatro a cinco meses.

A reserva estudada possuía, na época, áreas pouco alteradas e áreas que haviam sofrido exploração seletiva de componentes herbáceos e ou lenhosos, apresentando, portanto, locais com diferentes níveis de intervenção. Assim sendo, foram selecionadas três categorias de ambi- entes assim discriminadas: ambiente I - representado pelos locais onde o estrato inferior formado por Bromelia laciniosa Mart. ex Shult. (macambira) foi totalmente eliminado para ser fornecido ao gado durante os períodos de seca, nos últimos cinco anos. Além disso, este ambiente sofreu, também, exploração seletiva do estrato arbóreo para extração de estacas e lenha; ambiente II - caracterizado por um nível de perturbação menor que o anterior. Neste tipo de ambiente ocorreu a retirada do estrato inferior (Bromelia laciniosa), sem que tenha havido exploração significativa no estrato arbóreo; ambiente III - constituído por áreas do remanescente que se encontravam em melhor estado de conservação, tanto no que se refere ao estrato inferior quanto ao estrato arbóreo. Em cada ambiente foram plotadas, de forma aleatória, cinco parcelas de $2 \times 20 \mathrm{~m}$, nas quais foram feitas as avaliações. Os indivíduos presentes nas unidades amostrais foram classificados em quatro classes de tamanho de regeneração natural (CTRN), de acordo com Finol (1971) e adaptadas às condições de caatinga, conforme metodologia proposta por Rodal et al. (1992): classe 1 -indivíduos com altura variando de 0,10 a 0,29 m; classe 2-indivíduos com altura de 0,30 a 1,49 $\mathrm{m}$; classe 3-indivíduos com altura de 1,5 a $3 \mathrm{~m}$ e classe 4-indivíduos com altura superior a 3 $\mathrm{m}$, mas que apresentavam diâmetro ao nível do solo inferior a $3 \mathrm{~cm}$. Foram calculados os seguintes parâmetros: estimativa da densidade por classe de tamanho, freqüência, regeneração natural por classe de tamanho e regeneração total por espécie (Matteucci \& Colma, 1982; Mueller-Dombois \& Ellemberg, 1974; Braun-Blanquet, 1950).

\section{Resultados e Discussão}

Florística - No levantamento florístico foram identificados 347 indivíduos pertencentes a 17 famílias, 22 gêneros e 26 espécies. Tais valores mostraram-se superiores aos encontrados em outros trabalhos, realizados em ecossis- 
Tabela 1. Lista das famílias e espécies amostradas por ambiente (I = ambiente I; II = ambiente II e III = ambiente III), em ordem alfabética

\begin{tabular}{|c|c|c|c|c|}
\hline Família/Espécies & Nome vulgar & $\mathbf{I}$ & II & III \\
\hline $\begin{array}{l}\text { 1) Apocynaceae } \\
\text { 1- Aspidosperma pyrifolium Mart. }\end{array}$ & Pereiro & $\mathrm{x}$ & $\mathrm{x}$ & $\mathrm{x}$ \\
\hline $\begin{array}{l}\text { 2) Bignoniaceae } \\
\text { 2- Tabebuia impetiginosa (Mart. ex DC.) Standl. }\end{array}$ & Ipê-roxo & - & $\mathrm{x}$ & $\mathrm{x}$ \\
\hline $\begin{array}{l}\text { 3) Bombacaceae } \\
3 \text { - Pseudobombax sp. }\end{array}$ & Imbiratanha & - & - & $\mathrm{x}$ \\
\hline 4 - Ceiba glaziovii (Kuntze) K. Schum. & Barriguda & - & - & $\mathrm{x}$ \\
\hline $\begin{array}{l}\text { 4) Boraginaceae } \\
5 \text { - Cordia globosa (Jacq.) H.B.K }\end{array}$ & Moleque-duro & - & $\mathrm{x}$ & $\mathrm{x}$ \\
\hline $\begin{array}{l}\text { 5) Burseraceae } \\
6 \text { - Commiphora leptophloeos (Mart.) J. B Gillett. }\end{array}$ & Imburana- de-espinho & $\mathrm{x}$ & $\mathrm{x}$ & $\mathrm{x}$ \\
\hline $\begin{array}{l}\text { 6) Caesalpiniaceae } \\
7 \text { - Caesalpinia pyramidalis Tul. } \\
8 \text { - Bauhina cheilantha (Bong.) Steud. } \\
9 \text { - Caesalpinia ferrea Mart. ex Tul. } \\
7 \text { - Cannaraceae }\end{array}$ & $\begin{array}{l}\text { Catingueira } \\
\text { Mororó } \\
\text { Pau -ferro }\end{array}$ & $\begin{array}{l}x \\
- \\
x\end{array}$ & $\begin{array}{l}\mathrm{x} \\
\mathrm{x} \\
-\end{array}$ & $\begin{array}{l}\mathrm{x} \\
\mathrm{x} \\
-\end{array}$ \\
\hline $\begin{array}{l}\text { 7) Capparaceae } \\
10 \text { - Capparis cynophallophora L. } \\
11 \text { - Capparis jacobinae Moric. ex Eichl. }\end{array}$ & $\begin{array}{l}\text { Feijão-bravo } \\
\text { Icó }\end{array}$ & $\begin{array}{l}\mathrm{x} \\
\mathrm{x}\end{array}$ & - & - \\
\hline $\begin{array}{l}\text { 8) Combretaceae } \\
12 \text { - Thiloa glaucocarpa (Mart.) Eichl. }\end{array}$ & Sipaúba & $\mathrm{x}$ & $\mathrm{x}$ & $\mathrm{x}$ \\
\hline $\begin{array}{l}\text { 9) Euphorbiaceae } \\
13 \text { - Croton sonderianus Muell. Arg. } \\
14 \text { - Manihot graziovii Muell. Arg. } \\
15 \text { - Sapium sp }\end{array}$ & $\begin{array}{l}\text { Marmeleiro } \\
\text { Maniçoba } \\
\text { Burra-leiteira }\end{array}$ & $\begin{array}{l}\mathrm{x} \\
\mathrm{x} \\
\mathrm{x}\end{array}$ & $\begin{array}{l}\mathrm{x} \\
\mathrm{x} \\
-\end{array}$ & $\begin{array}{l}\mathrm{x} \\
\mathrm{x} \\
-\end{array}$ \\
\hline $\begin{array}{l}\text { 10) Fabaceae } \\
16 \text { - Amburana cearensis (Allem.) A. C. Smith }\end{array}$ & Imburana-de-cheiro & - & - & $\mathrm{x}$ \\
\hline $\begin{array}{l}\text { 11) Mimosaceae } \\
17 \text { - Piptadenia stipulacea (Benth) Ducke }\end{array}$ & Jurema-branca & $\mathrm{x}$ & $\mathrm{x}$ & $\mathrm{x}$ \\
\hline 18 - Mimosa cf. malacocentra Mart. & Amorosa & $\mathrm{x}$ & $\mathrm{x}$ & - \\
\hline $\begin{array}{l}19 \text { - Mimosa tenuiflora (Willd.) Poir. } \\
20 \text { - Acacia glomerosa Benth. }\end{array}$ & $\begin{array}{l}\text { Jurema -preta } \\
\text { Espinheiro }\end{array}$ & $\begin{array}{l}- \\
\mathrm{x}\end{array}$ & $\begin{array}{l}x \\
-\end{array}$ & - \\
\hline $\begin{array}{l}\text { 12) Myrtaceae } \\
21 \text { - Eugenia uvalha Cambess. }\end{array}$ & Ubaia & - & $\mathrm{x}$ & $\mathrm{x}$ \\
\hline $\begin{array}{l}\text { 13) Nictaginaceae } \\
22 \text { - Pisonia tomentosa Casar }\end{array}$ & João-mole & $\mathrm{x}$ & - & $\mathrm{x}$ \\
\hline $\begin{array}{l}\text { 14) Rhamnaceae } \\
23 \text { - Zizyphus joazeiro Mart. }\end{array}$ & Juazeiro & $\mathrm{x}$ & - & - \\
\hline $\begin{array}{l}\text { 15) Sapindaceae } \\
24 \text { - Allophylus sp. }\end{array}$ & Estraladeira & $\mathrm{x}$ & $\mathrm{x}$ & $\mathrm{x}$ \\
\hline $\begin{array}{l}\text { 16) Sterculiaceae } \\
25 \text { - Helicteres eichleri } \mathrm{K} \text {. Schum. }\end{array}$ & - & - & $\mathrm{x}$ & $\mathrm{x}$ \\
\hline $\begin{array}{l}\text { 17) Verbenaceae } \\
26 \text { - Lantana camara } \mathrm{L} .\end{array}$ & Chumbinho & $\mathrm{x}$ & $\mathrm{x}$ & $\mathrm{x}$ \\
\hline
\end{tabular}


temas de caatinga no Estado da Paraíba (Gomes, 1979), porém aproximam-se dos resultados obtidos por Ferreira (1988) e Araújo et al., (1995) em levantamentos florísticos realizados em condições similares. Tais diferenças são perfeitamente compreensíveis, considerando-se a heterogeneidade dos ecossistemas de caatinga e o caráter incipiente dos estudos desenvolvidos no referido bioma.

No ambiente I foram registrados 12 famílias, 16 gêneros e 18 espécies. As famílias que apresentaram maior número de espécies foram: Mimosaceae, com quatro; Euphorbiaceae e Caesalpiniaceae, com três espécies cada uma (Tab. 1). Juntas, essas famílias contribuíram com aproximadamente $56 \%$ do total de espécies amostradas neste ambiente. Estas espécies geralmente ocupam os primeiros lugares na maioria dos levantamentos florísticos realizados em ecossistemas de caatinga (Sampaio, 1996).

No ambiente II, foram amostradas 13 famílias, 16 gêneros e 17 espécies. As famílias Mimosaceae, com três espécies; Euphorbiaceae e Caesalpiniaceae, com duas espécies cada uma, detiveram cerca de $44 \%$ do total das espécies amostradas neste ambiente.

No ambiente III, foram encontradas 16 famílias, 19 gêneros e 19 espécies. As famílias que mais se destacaram em número de espécies foram: Euphorbiaceae e Caesalpiniaceae, com duas espécies cada uma (Tab. 1).

As espécies Croton sonderianus, Manihot glaziovii, Piptadenia stipulacea, Allophylus sp., Thiloa glaucocarpa, Capparis jacobinae, Lantana camara, Aspidosperma pyrifolium, Caesalpinia pyramidalis, e Commiphora leptophloe$o s$, estiveram presentes nos três ambientes. Observou-se, porém, que algumas espécies ocorreram apenas em um ou outro ambiente, dentre aqueles selecionados para estudo. Assim, as espécies Sapium sp., Acacia glomerosa, Caesalpinia ferrea, Capparis cynophallophora e Zizyphus joazeiro, foram exclusivas do ambiente I. Mimosa tenuiflora, ocorreu apenas no ambiente II e as espécies Amburana cearensis, Pseu- dobombax sp., e Ceiba glaziovii mostraram-se exclusivas do ambiente III (Tab. 1).

Considerando a extensão e a homogeneidade da área estudada, particularmente no que se refere às condições de relevo, de solos e de clima, não é de se esperar que existam fatores restritivos à ocorrência das espécies. Portanto, a distribuição destas por ambiente, conforme acima explicitada, pode ser entendida como uma resposta das mesmas à perturbação antrópica.

Distribuição das alturas - No ambiente I, cerca de $65 \%$ dos indivíduos apresentavam altura inferior a 1,5 $\mathrm{m}$ pertencendo, portanto, às classes de tamanho 1 e 2, com predomínio desta última. Os demais indivíduos, estavam compreendidos na classe de tamanho 3. No ambiente II, cerca de $98 \%$ dos indivíduos pertenciam às classes de tamanho $2 \mathrm{e} 3$, tendo-se percebido que algumas espécies presentes na regeneração natural já possuíam indivíduos aptos a completar todas as fases do ciclo vegetativo. $\mathrm{O}$ número de indivíduos registrado nestas duas classes foi bastante próximo, mostrando a evolução do processo de regeneração natural. Neste ambiente não foram registrados indivíduos pertencentes à quarta classe de tamanho, mas observou-se uma ligeira predominância da última classe sobre a primeira, indicando que provavelmente houve uma redução do nível de perturbação, em relação ao ambiente anterior. Já no ambiente III, formado pelas áreas em melhor estado de conservação, o número de indivíduos pertencentes à classe de tamanho 3 foi maior do que aquele encontrado nos ambientes anteriores (52\%). Constata-se aqui o surgimento de indivíduos na classe de tamanho 4, o que não foi registrado em nenhum dos outros ambientes. $\mathrm{O}$ ingresso dos indivíduos nas maiores classes de tamanho apresenta uma razão inversa ao nível de perturbação imputado aos ambientes. Esta constatação ratifica os resultados de Myster (1993), segundo os quais o estabelecimento das espécies se dá em razão inversa ao nível de perturbação sofrido pelos ambientes, entendendo-se estabelecimento como a ocorrên- 
cia de indivíduos capazes de completar todas a fases do ciclo vegetativo.

Densidade - As densidades totais obtidas neste trabalho foram de 6750,5500 e 5100 indivíduos $\mathrm{ha}^{-1}$, para os ambientes I, II e III, respectivamente (Fig. 1). A maior densidade apresentada no ambiente I, em relação aos demais, explica-se, provavelmente, pelo nível de perturbação a que o mesmo estava submetido. Esta hipótese é reforçada quando se analisa o comportamento de Croton sonderianus, cujos valores de densidade mostraramse inversamente proporcionais aos níveis de perturbação (Tab. 2 a 4). Esta tendência tem sido constatada, não apenas para esta espécie, mas também para outras pioneiras típicas de ambientes antropizados da caatinga, como demonstram os resultados obtidos por Sampaio et al., (1998); Sampaio, (1996) e Ferreira, (1988). Segundo Hardesty et al. (1988), o Croton sonderianus é uma espécie invasora de caatingas antropizadas, que produz grande quantidade de sementes, cuja dispersão acontece de forma fácil, tanto no momento da deiscência dos frutos, quanto posteriormente, através de vetores biológicos.

Tais características fazem da mesma uma pioneira típica da caatinga, que tende a dominar os primeiros estágios serais, aparecendo, portanto, como a espécie mais comum nas áreas sob grandes perturbações. Sampaio et al. (1998), após estudarem o efeito da perturbação (corte e queima) na vegetação de caatinga em Serra Talhada-PE, mostraram que estas práticas não apresentaram impactos negativos significativos sobre a espécie em questão. Estas informações ajudam a entender e ratificam os resultados obtidos neste trabalho.

No ambiente II, observa-se, o surgimento de novas espécies, apresentando uma densidade mais expressiva, como é o caso de

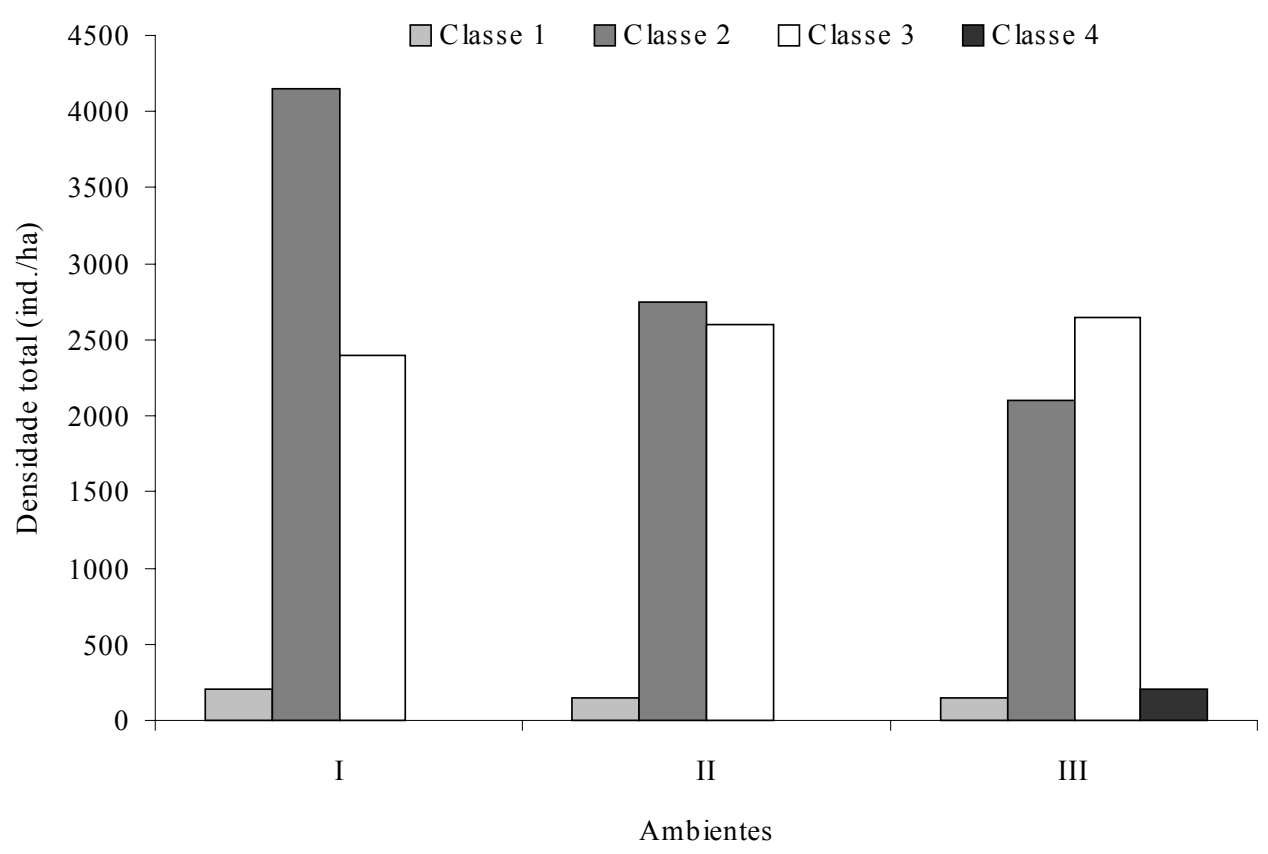

Figura 1. Distribuição da densidade por classe de tamanho da regeneração natural, sendo: Classe 1: 0,10 - $0,29 \mathrm{~m}$; Classe 2: 0,30 - 1,49 m; Classe 3: 1,50-3 m e Classe 4: altura superior a $3 \mathrm{~m}$ e diâmetro menor ou igual a $3 \mathrm{~cm}$. Ambiente I: maior grau de antropismo; Ambiente II: medianamente antropizado; Ambiente III: menor grau de antropismo. 
Cordia globosa, que não esteve presente no ambiente I, mas, apareceu no ambiente II, refletindo assim, uma diminuição do nível de perturbação. Esta espécie, segundo Sampaio et al. (1998), mostra-se bastante sensível à perturbação o que certamente limitou sua presença naquele tipo de ambiente. Outras espécies como Lantana camara e Tabebuia impetiginosa, também só surgiram a partir do ambiente II (Tab. 3).

No ambiente III as espécies que apresentaram os maiores valores de densidade, freqüência e regeneração natural total foram Thiloa glaucocarpa, seguida de Allophylus sp. A Thiloa glaucocarpa, de forma geral, apresentou-se bem distribuída em todo o remanescente, o que fora confirmado, pelos expressivos valores de IVI encontrados em levantamento fitossociológico realizado na mesma área por Pereira (2000).
O comportamento do Croton sonderianus nos três ambientes reflete, nitidamente, os efeitos das perturbações a que os mesmos estão submetidos. Esta tendência também foi manifestada por outras espécies, a exemplo de Piptadenia stipulacea e Mimosa cf. malacocentra. Estas espécies mostram-se bastante tolerantes a elevados níveis de perturbação (Sampaio et al., 1998), condições a que os ambientes I e II estão submetidos. Comportamento inverso foi apresentado por Allophylus sp. e Capparis jacobinae, que tiveram suas populações reduzidas à medida que o grau de antropismo dos ambientes aumentou.

$\mathrm{O}$ aumento da densidade dos indivíduos jovens é uma característica comum dos ecossistemas florestais antropizados (Bertoni, 1984). Vale salientar que o incremento desse parâmetro se dá pelo ingresso sazonal de indivíduos, muitos dos quais não atingirão a fase adulta devido à influência de inúmeros fato-

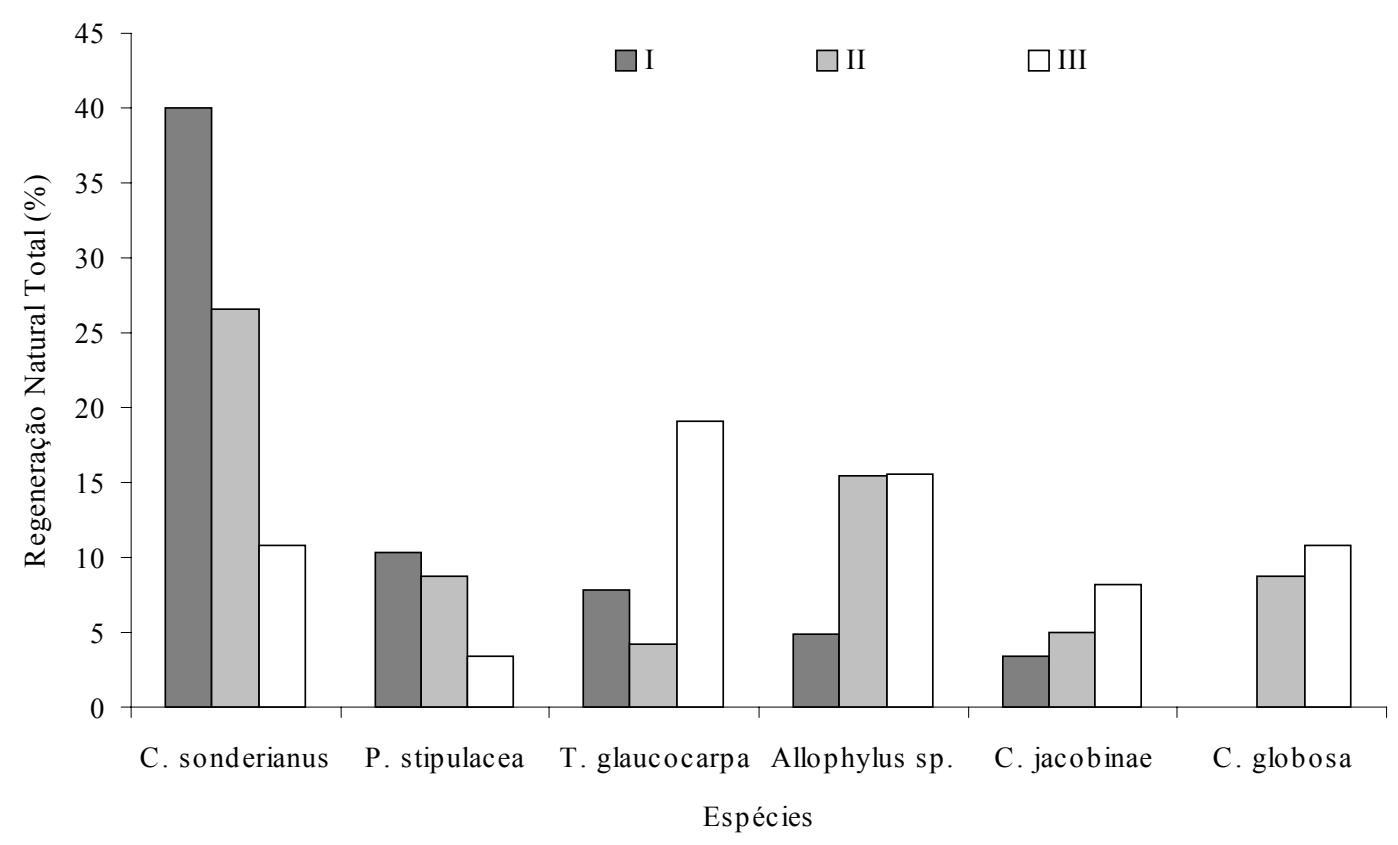

Figura 2. Comportamento das seis espécies de maior de regeneração natural total por ambiente 
res, tais como predação, competição, danos físicos, dentre outros (Fatubarin, 1987; Harmer, 1995).

Analisando-se de forma conjunta os dados relativos aos ambientes estudados, observa-se, com evidência, o efeito da ação antrópica sobre a vegetação. Este efeito manifesta-se tanto na concentração como na ausência de algumas espécies nos ambientes mais perturbados; na predominância de determinadas espécies ou no surgimento gradual de outras à medida que diminui a intensidade da perturbação (Fig. 2). Espécies como Croton sonderianus, Mimosa cf malacocentra e Capparis cynophallophora podem ser consideradas indicadoras do nível de perturbação antrópica, característica que se expressa pelo comportamento de suas populações nos ambientes avaliados (Tab. 1 a 4 ).

Conforme já fora dito, o fragmento estudado apresenta, em toda a sua extensão, as mesmas características de relevo, de solos e de clima. Em tais condições, não é de se esperar que existam fatores restritivos à ocorrência das espécies. A fisionomia da vegetação é muito uniforme e nada sugere a existência de sítios, cujas particularidades locais possam favorecer ou limitar a ocorrência das espécies em um ou outro ponto da reserva. Destaque-se ainda que as ações antrópicas são efetuadas aleatoriamente ou seguindo critérios de acesso que facilitem o escoamento dos produtos explorados. No caso particular, estas ações foram realizadas em diversos pontos da reserva e em alguns dos quais foram plotadas as unidades amostrais. Portanto, os resultados obtidos indicam que as diferenças florístico-fitossociológicas, apresentadas pelos ambientes, podem ser atribuídas às ações antrópicas localmente constatadas.

Conclui-se que os níveis de perturbação antrópica, conforme caracterizados neste trabalho, afetaram diretamente o processo de regeneração natural. Esta influência se revela de forma mais objetiva sobre a densidade e a distribuição das espécies nas classes de altura e na composição florística. As alterações da vegetação foram diretamente proporcionais ao nível de perturbação a que os ambientes haviam sido submetidos, sendo possível caracterizar algumas espécies como indicadoras da intensidade da perturbação, a exemplo de Croton sonderianus, Mimosa cf. malacocentra, Capparis cynophalophora, Amburana cearensis, Pseudobombax sp. e Ceiba glaziovii. 


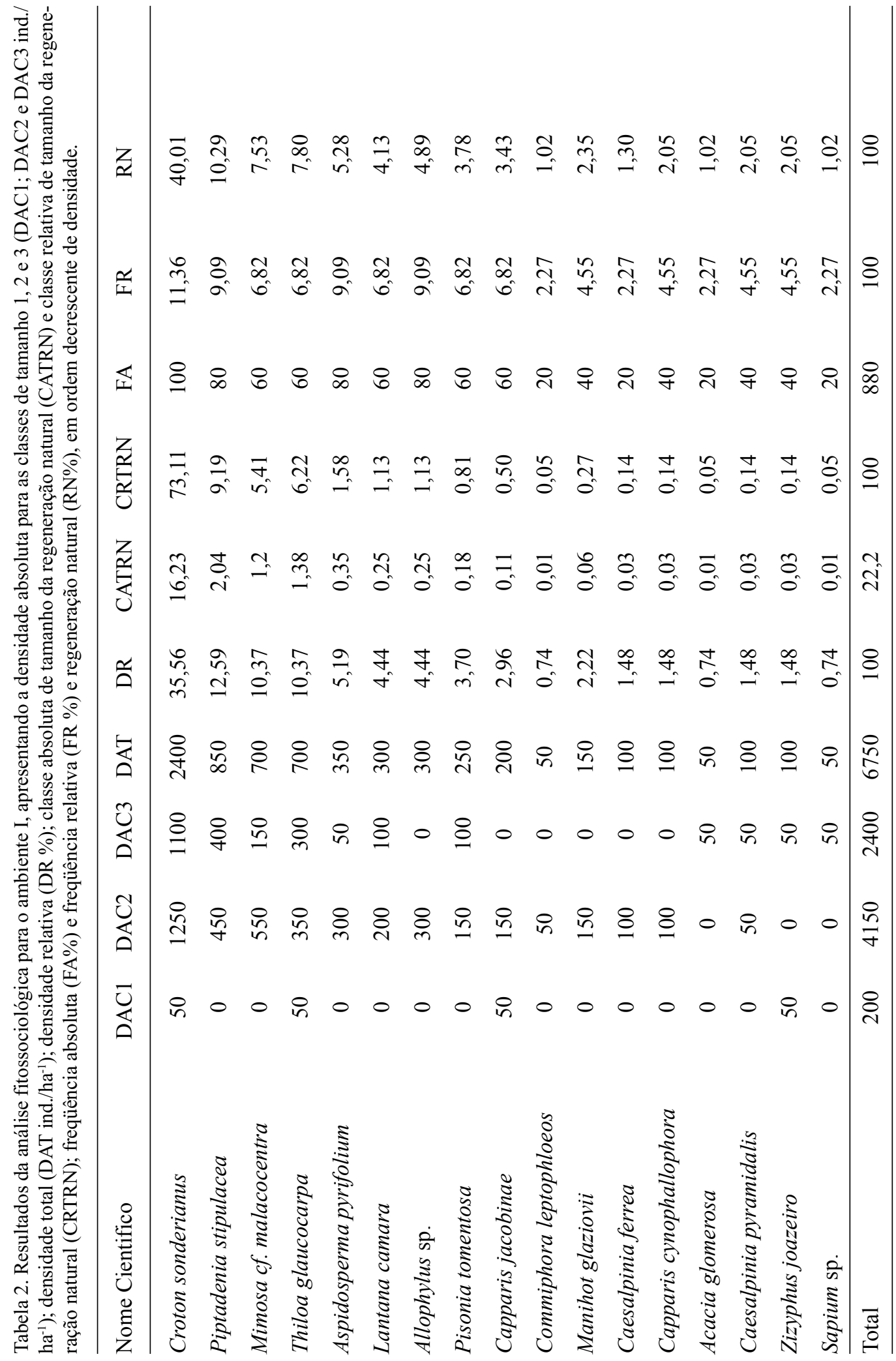




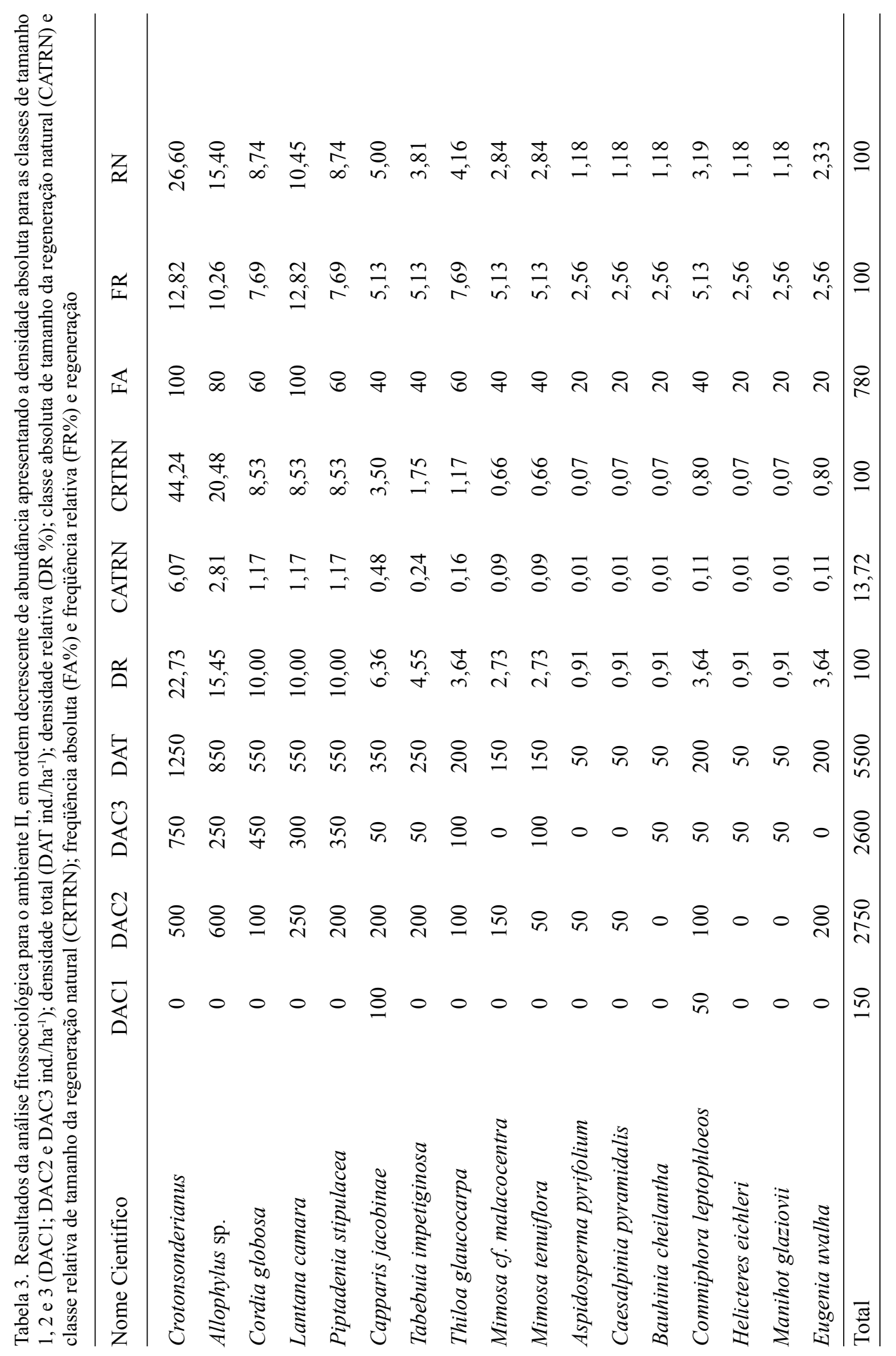


论

ปิ

\begin{tabular}{|c|c|c|c|c|c|c|c|c|c|c|c|c|c|c|c|c|c|c|c|c|c|}
\hline & $\stackrel{0}{a}$ & $\begin{array}{l}n \\
n \\
n\end{array}$ & $\stackrel{n}{\tilde{0}}$ & $\stackrel{n}{\circ}$ & $\stackrel{\Xi}{\infty}$ & $\stackrel{\infty}{+}$ & & $\begin{array}{l}\infty \\
\infty \\
+ \\
+\end{array}$ & ले & $\begin{array}{l}\tilde{\infty} \\
i\end{array}$ & $\begin{array}{l}\overrightarrow{6} \\
m\end{array}$ & $\begin{array}{l}\infty \\
\infty \\
i\end{array}$ & $\stackrel{0}{n}$ & $\stackrel{\vec{m}}{i}$ & $\stackrel{\vec{m}}{\mathrm{i}}$ & $\stackrel{\circ}{\sim}$ & $\stackrel{0}{\sim}$ & $\Xi$ & $\Xi$ & $\Xi$ & $\Xi$ \\
\hline & $\begin{array}{l}\hat{6} \\
=\end{array}$ & $\begin{array}{l}\text { స్ } \\
\text { aे }\end{array}$ & ๙ి & ñ & 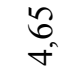 & $\begin{array}{l}n \\
+ \\
+\end{array}$ & & مَ & \begin{tabular}{l}
$\tilde{b}$ \\
\multirow{\sigma}{*}{}
\end{tabular} & 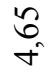 & $\begin{array}{l}\infty \\
\hat{\sigma}\end{array}$ & 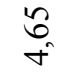 & $\begin{array}{l}n \\
\stackrel{n}{N}\end{array}$ & $\begin{array}{l}6 \\
8 \\
8\end{array}$ & $\begin{array}{l}6 \\
\forall \\
\forall\end{array}$ & $\stackrel{m}{\tilde{i}}$ & $\stackrel{m}{\hat{n}}$ & $\stackrel{m}{\sim}$ & $\stackrel{m}{\sim}$ & $\stackrel{m}{\stackrel{n}{i}}$ & $\Xi$ \\
\hline & $\begin{array}{l}8 \\
8 \\
8\end{array}$ & $\begin{array}{l}8 \\
8 \\
\infty\end{array}$ & $\begin{array}{l}8 \\
8 \\
\infty\end{array}$ & $\begin{array}{l}8 \\
\infty \\
\infty\end{array}$ & $\begin{array}{l}8 \\
8 \\
8\end{array}$ & $\begin{array}{l}8 \\
8 \\
\end{array}$ & ? & $\begin{array}{l}8 \\
8 \\
8 \\
8\end{array}$ & \begin{tabular}{l}
8 \\
$\stackrel{\circ}{+}$ \\
\multirow{+}{*}{}
\end{tabular} & $\begin{array}{l}8 \\
8 \\
8\end{array}$ & $\begin{array}{l}8 \\
8 \\
8\end{array}$ & $\begin{array}{l}8 \\
8 \\
8\end{array}$ & $\begin{array}{l}8 \\
\text { ¿ } \\
\text { i }\end{array}$ & $\begin{array}{l}8 \\
8 \\
8\end{array}$ & $\begin{array}{l}8 \\
8 \\
8\end{array}$ & $\begin{array}{l}8 \\
\text { in }\end{array}$ & $\begin{array}{l}8 \\
\stackrel{\text { Dे }}{ }\end{array}$ & $\begin{array}{l}8 \\
\stackrel{\ominus}{0}\end{array}$ & $\begin{array}{l}8 \\
\stackrel{\sim}{\circ}\end{array}$ & 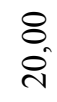 & 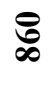 \\
\hline & $\begin{array}{l}\text { ठे } \\
\text { নे }\end{array}$ & 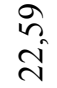 & $\stackrel{n}{\simeq}$ & $\frac{n}{1}$ & $\begin{array}{l}\text { J } \\
0 \\
0\end{array}$ & $\stackrel{\text { S }}{+}$ & & हn & $\stackrel{\overrightarrow{6}}{-}$ & ̊. & مे & o̊ & $\stackrel{+}{+}$ & $\stackrel{\circ}{+}$ & $\stackrel{+}{+}$ & $\stackrel{o}{+}$ & 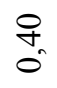 & $\frac{0}{0}$ & $\stackrel{0}{0}$ & $\frac{0}{0}$ & $\Theta$ \\
\hline & $\begin{array}{l}\infty \\
\infty \\
i\end{array}$ & $\vec{\sim}$ & $\stackrel{9}{=}$ & $\stackrel{9}{=}$ & $\stackrel{\infty}{\circ}$ & $\stackrel{\infty}{0}$ & & $\begin{array}{l}\hat{y} \\
\tilde{o}\end{array}$ & $\frac{6}{0}$ & $\hat{o}_{0}$ & $\hat{\sigma}_{0}$ & oे & $\begin{array}{l}\text { Oे } \\
0\end{array}$ & $\begin{array}{l}\text { I } \\
0\end{array}$ & $\begin{array}{l}\text { U } \\
0\end{array}$ & $\begin{array}{l}\text { J } \\
0 \\
0\end{array}$ & $\stackrel{0}{0}_{0}^{t}$ & $\overline{0}$ & $\bar{\sigma}_{0}$ & $\begin{array}{l}0 \\
0 \\
0\end{array}$ & $\frac{2}{a}$ \\
\hline & $\begin{array}{l}\hat{\sigma} \\
\hat{\sigma}^{-}\end{array}$ & $\underset{\Xi}{\nexists}$ & $\stackrel{\infty}{\stackrel{\infty}{0}}$ & $\stackrel{\infty}{\stackrel{\infty}{0}}$ & $\begin{array}{l}\infty \\
\sigma\end{array}$ & $\begin{array}{l}0 \\
0 \\
0\end{array}$ & & 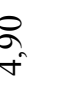 & 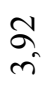 & $\stackrel{+}{\text { dे }}$ & $\begin{array}{l}\text { वे } \\
\text { iे }\end{array}$ & ते & $\stackrel{\circ}{-}$ & $\stackrel{\circ}{-}$ & $\stackrel{\circ}{\Im}$ & $\stackrel{2}{-}$ & $\stackrel{\circ}{\circ}$ & $\stackrel{\infty}{\sigma}$ & $\stackrel{\infty}{\sigma}$ & $\stackrel{\infty}{\sigma}$ & $\Xi$ \\
\hline & $\stackrel{8}{n}$ & $\stackrel{\circ}{n}$ & in & in & in & $\stackrel{\circ}{n}$ & & $\begin{array}{l}0 \\
i\end{array}$ & $\stackrel{\text { ¿ }}{\text { ¿ }}$ & $\stackrel{\circ}{n}$ & $\stackrel{ }{n}$ & $\stackrel{\circ}{n}$ & ○ & 8 & 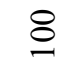 & $\cong$ & 8 & in & in & in & $\frac{8}{8}$ \\
\hline & 0 & 0 & 0 & 0 & 0 & 0 & $c$ & 0 & $\stackrel{n}{n}$ & 0 & 0 & 0 & in & 0 & 0 & 0 & 0 & 0 & 0 & 0 & ஓ् \\
\hline & ஜn & in & \& & \& & in & 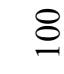 & & in & in & 0 & 8 & in & in & 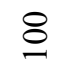 & in & 0 & in & 0 & in & in & : \\
\hline & $\stackrel{\sim}{\stackrel{i}{ }}$ & ஓ্ণ & $\stackrel{\circ}{n}$ & in & \&্ల & 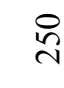 & 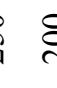 & 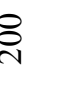 & 0 & 은 & in & 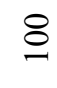 & 0 & 0 & in & 8 & in & in & 0 & 0 & $\stackrel{\text { ㄱ }}{\mathrm{N}}$ \\
\hline & 0 & 0 & 0 & 0 & 으 & 0 & & 0 & 0 & 0 & 0 & 0 & 0 & 0 & 0 & 0 & 0 & 0 & 0 & 0 & 17 \\
\hline 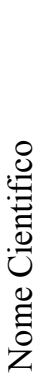 & 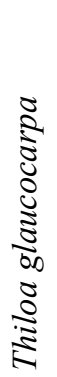 & 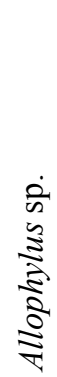 & $\begin{array}{l}0 \\
0 \\
0 \\
0 \\
: 0 \\
0 \\
0 \\
0\end{array}$ & 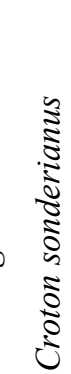 & 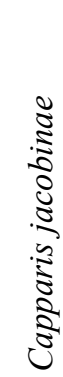 & 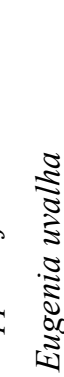 & 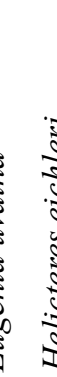 & 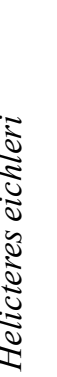 & 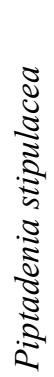 & 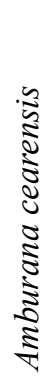 & 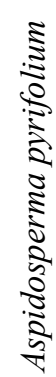 & 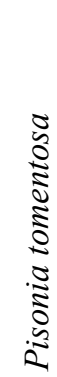 & 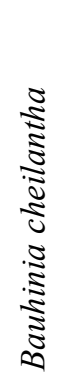 & 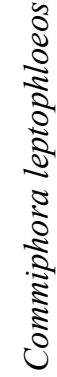 & 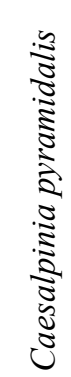 & 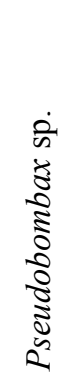 & 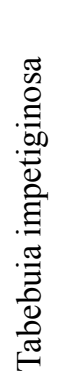 & 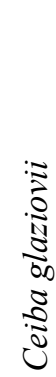 & 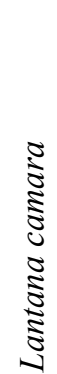 & 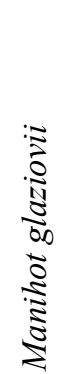 & $\stackrel{\bar{\pi}}{\stackrel{\tilde{\theta}}{\theta}}$ \\
\hline
\end{tabular}




\section{Referências Bibliográficas}

Ab'Saber, A. N. 1977. Problemática da Desertificação e da Savanização no Brasil Intertropical. São Paulo. EDUSP, Instituto de Geografia, pp. 1-19.

Albuquerque, S. G. de. 1999. Caatinga vegetation dynamics under various grazing intensities by steers in the semi-arid Northeast, Brazil. Journal of Range Management 52: 241-248.

Andrade, L. A.; Reis, M. G.; Reis, G. G. 1999. Classificação ecológica do Estado da Paraíba. Interpolação de dados climáticos por aproximação numérica. Revista Árvore 23 (1): 23-32.

Andrade-Lima, D. 1981. The caatingas dominium. Revista Brasileira de Botânica 4 (2): 149-153.

Araújo Filho, J. A. 1996. Desenvolvimento Sustentável da Caatinga. Sobral: Ministério da Agricultura/ EMBRAPA/CNPC, 20p.

Araújo, E.L.; Sampaio, E. V. S. B.; Rodal, M .J. N. 1995. Composição florística e fitossociológica de três áreas de caatinga em Pernambuco. Revista Brasileira de Biologia 55(4): 595-607.

Bertoni, J. E. A. 1984. Composição florística e estrutura de uma floresta do interior do Estado de São Paulo: Reserva Estadual de Porto Ferreira. Dissertação de Mestrado. Universidade Estadual de Campinas, Campinas.

BRASIL. Ministério da Agricultura. Equipe de Pedologia e Fertilidade do Solo. Divisão de agrologia - SUDENE. 1971. Levantamento Exploratório. Reconhecimento de solos do Estado da Paraíba. Rio de Janeiro: 670p. (Boletim técnico, 15).

BRASIL, Ministério das Relações Exteriores. CIMA. 1991. Subsídios Técnicos para elaboração do relatório nacional do Brasil para a CNUMAD. Brasília. 172p.

BRASIL, Secretaria de Planejamento, Orçamento e Coordenação. Fundação Instituto Brasileiro de Geografia e Estatística. 1992. Manual Técnico da Vegetação Brasileira. Rio de Janeiro. 92p. (Série Manuais Técnicos em Geociências, 1).

BRASIL, Ministério do Planejamento e Orçamento. 1995. Nordeste: uma estratégia de desenvolvimento sustentável. Brasília. 231 p.

Braun-Blanquet, J. 1950. Sociologia vegetal: estudio de las comunidades vegetales. Buenos Aires: Acme, 44p.

Calegario, N.; Souza, A. L.; Marangon, L. C.; Silva, A. F. da. 1993. Parâmetros florísticos e fitossociológicos da regeneração natural de espécies arbóreas nativas no sub-bosque de povoamentos de Eucalyptus. Revista Árvore 17(1):19-29,

Daubenmire, R. 1968. Plant Communities. A text book of plant synicology. Harper \& Row, Publishers. New York, 293p.

Dregne, H. E. 1986. Desertification of Arid Lands. Hardwood Academy (Coleção: Advances in Desert and Arid Land Technology and development, 3). s.1.

Drumond, M. A.; Barros, N. F. Souza, A. L.; Silva, A. F. Meira Neto, J. A. A. 1996. Alterações Fitossociológicas e edáficas na mata atlântica em função das modificações da cobertura vegetal. Revista Árvore 20 (4).

Fatubarin, A. 1987. Observations on the natural regeneration of the woody plants in a savana ecosystem in Nigeria. Tropical Ecology 28:1-8.

Ferreira, R. 1988. Análise estrutural da vegetação da Estação Florestal de Experimentação de Açú RN, como subsídio básico para o manejo florestal. Dissertação de Mestrado, Universidade Federal de Viçosa, Viçosa.

Finol, U. H. 1971. Nuevos parâmetros a considerarse en el analisis estrutural de las selvas virgenes tropicales. Revista Florestal Venezuelana 14 (21):29-42.

Gomes, M. A. F. 1979. Padrões de caatinga nos Cariris Velhos, Paraíba. Dissertação de Mestrado. Universidade Federal Rural de Pernambuco, Recife.

Hardesty, L .H.; Box, T.W.; Malechek, J. C. 1988. Season of cutting affects biomass production by coppicing browse species of the Brazilian caatinga. Journal of Range Management 41(6): 477 480.

Harmer, R. 1995. Natural regeneration of broadleaved trees in Britain: III germination and establishment. Forestry 68(1):1-9.

IBAMA. 1992. Plano de manejo florestal para a região do Seridó do Rio Grande do Norte. Natal: IBAMA, v.1. (Projeto PNUD/FAO/IBAMA).

Japan, Environment Agency. 1990. Global Enviroment Program and Global Enviroment Monitoring Program for Fiscal Year 1990. Enviroment Agency. Tokio.

Matteucci, S. D.; Colma, A. 1982. Metodologia para el estudio de la vegetacion. Programa Regional de Desarrolo Científico y Tecnológico. Washington. $168 \mathrm{p}$.

Mcboyle, G. R. 1973. Climate classification of Australia by computer. In: McBOYLE, G., ed. Climate in review. Houghton Mifflin. Boston.

Mueller-Dombois, D.; Ellemberg, H. 1974. Aims and methods of vegetation ecology. New York, John 
Wiley \& Sons, $547 \mathrm{p}$.

Myster, R. W. 1993.Tree Invasion and Establishment in Old Fields at Hutcheson Memorial Forest. The Botanical Review. 59(4): 251-272.

Nimer, E. 1972. Climatologia da Região Nordeste do Brasil. Subsídios à Geog. Reg. do Brasil. Revista Brasileira de Geografia, 34 (2):3-51.

Pereira, I. M. 2000. Levantamento florístico do estrato arbustivo-arboreo e análise da estrutura fitossociológica de ecossistema de caatinga sob diferentes níveis de antropismo. Dissertação de Mestrado. Universidade Federal da Paraíba, Areia.

Reddy, S.J. 1983. Climatic classification: the semi-arid tropics and its environment - a review. Pesquisa Agropecuária Brasileira 18 (8): 823-847.

Rodal, M. J. N.; Sampaio, E. V. S. B.; Figueiredo. 1992. M. A. Manual sobre métodos de estudo florístico e fitossociológico - ecossistema de caatinga. Sociedade Botânica do Brasil. Brasília.

Sampaio, E. V. S. B, Salcedo, I. H. 1993. Effect of different fire severities on coppicing of caatinga vegetation in Serra Talhada, PE, Brazil. CORVALLIS-OREGON-USA, 1993. Biotropica 25 (4): 452-460.

Sampaio, E. V. S. B. 1995. Overview of the Brazilian Caatinga. In.: Bullock, S. H. Mooney; Medina, E. Seasonally dry tropical forests. Cambrige University Press.

Sampaio, E.V. S. B. 1996. Fitossociologia. In: Sampaio, E. V. S. B.; Mayo, S. J \& Barbossa, M. R. V. (Eds.). Pesquisa Botânica do Nordeste: Progresso e perspectivas. Recife: Sociedade Botânica do Brasil.

Sampaio, E. V. S. B., Araújo, E.L. de., Salcedo, I. H. \& Tiessen, H. 1998. Regeneração da vegetação de Caatinga após corte e queima, em Serra Talhada, PE. Pesquisa Agropecuária Brasileira 33 (5): 621-632.

Sousa, J. G. de. 1979. O Nordeste brasileiro: uma experiência de desenvolvimento regional. Fortaleza: Banco do Nordeste do Brasil, Fundação Getúlio Vargas. 410p. 